\title{
Avaliação da hiperlactatemia em pacientes com diagnóstico de choque séptico
}

Evaluation of hyperlactatemia in patients diagnosed with septic shock

Evaluación de la hiperlactatemia en pacientes diagnosticados de shock séptico

Júlia Domingues Gatti ${ }^{1 *}$, Aline Vieira Guimarães ${ }^{1}$, Aline Vilela de Oliveira Paranaíba ${ }^{2}$, Flávia Ferrazzo $^{2}$, Isabella Orlc Figueiredo Melo', Larissa Giacomelli Stavro², Letícia Freire Rettore Mendes$^{1}$, Victória Gabriela Marcon², Vitória Miranda Thomaz², Mônica Isaura Corrêa ${ }^{3}$.

\section{RESUMO}

Objetivo: Realizar uma revisão da literatura acerca de como o aumento do lactato arterial pode influenciar no prognóstico dos pacientes em quadro de choque séptico. Revisão bibliográfica: $O$ choque séptico é definido como uma condição clínica em que o paciente apresenta a necessidade de vasopressor para manter uma pressão arterial média (PAM) de $65 \mathrm{mmHg}$ ou mais, e o nível de lactato sérico se encontra maior que $2 \mathrm{mmol} / \mathrm{L}$. O lactato é produzido devido a uma resposta de hipoperfusão tecidual, através do metabolismo anaeróbio, e em condições fisiológicas anormais é um composto utilizado como parâmetro do equilíbrio de oxigênio. Além disso, a redução em $10 \%$ no valor do lactato é responsável por diminuir cerca de $11 \%$ na taxa de mortalidade. Considerações finais: $O$ lactato arterial elevado mostrou-se relacionado ao aumento da taxa de mortalidade, ou seja, quanto maior o nível do composto pior é o prognóstico. Para isso, o diagnóstico precoce e medidas terapêuticas rapidamente instruídas são necessárias para aumentar a taxa de sobrevida dos pacientes em choque séptico.

Palavras-chave: Ácido láctico, Choque séptico, Prognóstico.

\begin{abstract}
Objective: Conduct a review the literature on how the increase in arterial lactate can influence the prognosis of patients with septic shock. Bibliographic review: Septic shock is defined as a clinical condition in which the patient needs a vasopressor to maintain an average arterial pressure (MAP) of $65 \mathrm{mmHg}$ or more, and the serum lactate level is greater than $2 \mathrm{mmol} / \mathrm{L}$. Lactate is produced due to a tissue hypoperfusion response, through anaerobic metabolism and under abnormal physiological conditions, it is a compound used as an oxygen balance parameter. In addition, the $10 \%$ reduction in the value of lactate is responsible for decreasing about $11 \%$ in the mortality rate. Final considerations: High arterial lactate was shown to be related to the increase in the mortality rate, that is, the higher the level of the compound, the worse the prognosis. For this, early diagnosis and promptly instructed therapeutic measures are necessary to increase the survival rate of patients in septic shock.
\end{abstract}

Key words: Lactic acid, Shock septic, Prognosis.

\section{RESUMEN}

Objetivo: Realizar una revisión la literatura sobre cómo el aumento del lactato arterial puede influir en el pronóstico de los pacientes con shock séptico. Revisión bibliográfica: El choque séptico se define como una

\footnotetext{
${ }^{1}$ Faculdade de Medicina de Barbacena (FAME), Barbacena - MG. *E-mail:juliadgatti@gmail.com

2 Universidade Cidade de São Paulo (UNICID), São Paulo - SP.

${ }^{3}$ Faculdade de Medicina do Vale do Aço (UNIVAÇO), Ipatinga - MG.
} 
condición clínica en la que el paciente necesita un vasopresor para mantener una presión arterial promedio (PAM) de $65 \mathrm{mmHg}$ o más, y el nivel de lactato sérico es mayor de $2 \mathrm{mmol} / \mathrm{L}$. El lactato se produce debido a una respuesta de hipoperfusión tisular, a través del metabolismo anaeróbico y en condiciones fisiológicas anormales, es un compuesto utilizado como parámetro de equilibrio de oxígeno. Además, la reducción del $10 \%$ en el valor del lactato es responsable de disminuir aproximadamente un $11 \%$ en la tasa de mortalidad. Consideraciones finales: Se demostró que el lactato arterial alto está relacionado con el aumento de la tasa de mortalidad, es decir, cuanto mayor es el nivel del compuesto, peor es el pronóstico. Para ello, es necesario un diagnóstico precoz y medidas terapéuticas instruidas con prontitud para aumentar la tasa de supervivencia de los pacientes en choque séptico.

Palabras clave: Ácido láctico, Choque séptico, Prognosis.

\section{INTRODUÇÃO}

A sepse representa um grande desafio para a saúde pública, levando milhares de pessoas à morte por ano, além de propiciar elevados custos financeiros para as instituições de saúde públicas e pacientes. Sua incidência vem aumentando bastante nos últimos anos, variando entre países e continentes (BARROS LL, et al., 2016). O choque séptico (CS) é uma classe da sepse em que o sistema circulatório e as anormalidades celulares e metabólicas estão associadas a um risco maior de mortalidade do que a sepse isolada (ARMSTROMG BA, et al., 2017).

O CS se dá pela ativação do sistema imune, além dos distúrbios causados na microcirculação durante o quadro de sepse, desempenhando um papel importante na elevação dos níveis de lactato. Tais fatores vão alterar o metabolismo da fosforilação oxidativa, visto que a quantidade de oxigênio que é fornecido às células se torna insuficiente (KHODASHAHI R e SARJAMEE S, 2020; BAKKER J, et al., 2019).

Devido à essa diminuição da oferta de oxigênio para os tecidos, ocorre uma transição no metabolismo da glicose em que a produção que era feita através do ciclo de Krebs, passa a ser produzido pela via anaeróbica, ou seja, a via do lactato. Esta última via produz apenas oito moléculas de ATP, enquanto a glicólise aeróbia produz cerca de 36 moléculas de ATP, e além disso, é caracterizada pela produção de ácido lático. O nível de lactato sanguíneo reflete diretamente no tempo de duração da hipóxia tecidual (JOUFFROY R, et al., 2020).

Em condições fisiológicas anormais, tem-se como marcador do equilíbrio de oxigênio, o aumento dos níveis de lactato (BAKKER J, et al., 2019; KHODASHAHI R E SARJAMEE S, 2020). O lactato é conhecido por desempenhar um papel importante na produção de energia e no metabolismo celular. A acidose láctica pode ocorrer em várias condições, como na sepse e choque séptico (LEE SG, et al., 2021). Para melhor avaliação do quadro séptico, a medida do lactato sérico inicial é aceita como método de avaliação da perfusão tecidual nos pacientes. Além disso, pode ser utilizada como triagem, sendo um parâmetro para início de condutas de ressuscitação volêmica (CHEN H, et al., 2021).

A normalização do lactato após medidas terapêuticas é um conceito conhecido para a previsão de melhores resultados no CS. Evidências mostraram que a melhora em cerca de $10 \%$ da depuração de lactato foi responsável por diminuir a taxa de mortalidade em $11 \%$ dos pacientes com diagnóstico de CS (KHODASHAHI R e SARJAMEE S, 2020). Além do mais, a adequação de parâmetros de perfusão tecidual, como a oligúria, livedo reticular e mottling score são normalizados após redução acentuada dos níveis de lactato (BAKKER J, et al., 2019).

No Brasil, existem várias razões para que a mortalidade da sepse e CS sejam elevadas, e particularmente, na rede pública. A carência dos leitos nas unidades de terapia intensiva, o desconhecimento dos profissionais de saúde sobre os sinais de alerta e gravidade, associados ao quadro infeccioso, fazem com que os pacientes tenham um diagnóstico tardio. Assim sendo, o tratamento deve ser feito o mais rápido possível para evitar que ocorra comprometimento sistêmico, e consequentemente, aumento da sobrevida do paciente (ILAS, 2015). 
Diante do exposto, o objetivo do presente trabalho é fazer uma revisão da literatura narrativa acerca de como o aumento do lactato arterial pode influenciar no prognóstico dos pacientes em quadro de choque séptico.

\section{REVISÃO BIBLIOGRÁFICA}

\section{Choque Séptico}

O CS é definido como uma condição clínica em que o paciente apresenta a necessidade de vasopressor para manter uma pressão arterial média (PAM) de $65 \mathrm{mmHg}$ ou mais, e o nível de lactato sérico se encontra maior que $2 \mathrm{mmol} / \mathrm{L}$. Dados estes, utilizados nas primeiras horas de ressuscitação volêmica para avaliação da resposta do paciente (RODRíGUEZ A, et al., 2016).

Embora os mecanismos envolvidos no CS sejam de extrema complexidade, a hipoperfusão tecidual contribui para a resposta inflamatória, levando a uma ativação endotelial, vasodilatação, liberação de mediadores inflamatórios e modulação do sistema de coagulação, sendo estas alterações responsáveis pela disfunção orgânica e óbito dos pacientes (ILAS, 2015).

A produção do lactato ocorre devido à diminuição da oferta de oxigênio para as células e tecidos em resposta a uma infecção causada por um hospedeiro. Dessa forma, a produção de lactato através do piruvato e catalisado pela desidrogenase lática (LDH) vai aumentar de maneira significativa, e assim, servirá como fonte de energia devido ao metabolismo anaeróbico, ou seja, sem a presença de oxigênio (YANG $\mathrm{H}$, et al., 2020).

A utilização do lactato como um marcador fisiológico do equilíbrio de oxigênio, como o nível inicial ou sua depuração, são dados utilizados como valor prognóstico de mortalidade em pacientes diagnosticados com CS. Além disso, o envelhecimento é um fator de risco, sendo os idosos mais propensos a evoluírem para óbito quando comparado aos pacientes mais jovens (KHODASHAHI R e SARJAMEE S, 2020).

$\mathrm{O}$ aumento do lactato tem sido tradicionalmente considerado um sinal de hipoperfusão tecidual/hipóxia nos pacientes em CS (CASTRO R, et al., 2020). Embora os estudos de Lokhandwala S, et al. (2016) mostrem que os pacientes com sepse e choque séptico apresentam aumento do lactato arterial, por mais que a fonte de elevação de lactato seja considerada, por vários autores, um sinal de hipoperfusão, neste estudo a etiologia exata ainda é discutida, visto que concluíram que a origem pode estar relacionada a lesão celular/tecidual ou ser uma causa multifatorial.

Pacientes em CS requerem cuidados intensivos por dias e até mesmo semanas, sendo o manejo hemodinâmico de extrema importância para as abordagens diagnósticas e terapêuticas, a fim de identificar e resolver alterações no sistema circulatório. Isso inclui cuidados desde o diagnóstico diferencial até a ressuscitação volêmica precoce e terapia hemodinâmica de pacientes com CS associado a complicações mais complexas, como a síndrome do desconforto respiratório agudo (SDRA), insuficiência renal, síndrome compartimental abdominal ou até mesmo a disfunção miocárdica preexistente (SAUGEL B, et al., 2016).

Além disso, o diagnóstico do CS/sepse é realizado através da detecção de patógenos por meio de culturas bacterianas e índices bioquímicos, que além do lactato, tem-se a procalcitonina (PCT), pró-hormônio da calcitonina, e a proteína $C$ reativa (PCR), considerada uma proteína de fase aguda produzida pelo fígado. São marcadoras bioquímicas que vêm mostrando bons resultados no diagnóstico e servindo de orientação para o manejo de antibióticos. Estudos evidenciam que as alterações nas concentrações de PCT e PCR estão relacionadas com o prognóstico dos pacientes (CUI NA, et al., 2019)

\section{Metabolismo do lactato}

O sistema cardiorrespiratório possui como função, a estabilidade e constância de oferta de oxigênio às células, propiciando o necessário à demanda metabólica do organismo, ou seja, a energia requerida para seu funcionamento. Nas situações patológicas, essa demanda metabólica pode não ser atendida, uma vez que o sistema cardiorrespiratório fica incapaz de fornecer quantidade adequada de oxigênio para as células e tecidos (ILAS, 2015). 
Depois de captado da atmosfera, o oxigênio difunde-se para o espaço alveolar dos pulmões. Após isso, cai na corrente sanguínea, no sangue capilar, onde é carreado pela proteína hemoglobina e uma pequena parte fica dissolvida no plasma. Para alcançar o espaço intracelular, o oxigênio é liberado pela hemoglobina. Dentro da célula, por meio do ciclo de Krebs e da cadeia respiratória, é transformado em energia com fins de manter as funções celulares vitais (KHODASHAHI R e SARJAMEE S, 2020).

Segundo Jouffroy R, et al. (2020), em condições patológicas, ocorre prejuízo na captação do oxigênio para os tecidos, causando hipóxia. No CS, devido à hipóxia tecidual e o consequente aumento de glicólise anaeróbia para suprir a oferta celular energética, ocorre aumento dos níveis de lactato. Normalmente, produzse no músculo esquelético, intestino, cérebro e hemácias, uma taxa de $1 \mathrm{mmol} / \mathrm{kg} / \mathrm{h}$ de lactato.

A partir da glicólise anaeróbia, o lactato então é produzido no citoplasma a partir da molécula piruvato. Por intermediar diversas reações do metabolismo, o piruvato sofre ação de várias enzimas. O complexo piruvatodesidrogenase o transforma em acetilcoenzima $A$, que será condensado com o oxaloacetato, gerando citrato no ciclo de Krebs. A enzima piruvato-carboxilase o converte a oxaloacetato, a alanina-aminotransferase 0 converte a alanina e a lactato-desidrogenase o converte a lactato. No citoplasma, a reação do piruvato catalisado pela enzima LDH tende a manter concentrações iguais de piruvato e lactato. Portanto, o aumento da concentração citoplasmática de lactato é proporcional ao aumento de concentração citoplasmática do piruvato (FURLAN JP, et al., 2019; JOUFFROY R, et al., 2019).

$\mathrm{O}$ aumento das concentrações de lactato é responsável pela queda do $\mathrm{pH}$ sanguíneo e pela produção dos íons de $\mathrm{H}_{+}$, consequentemente, ocorre aumento do tamponamento pelo bicarbonato de sódio, que tem como produto final, água e CO2. Dessa forma, ocorre relação de causa e efeito entre o aumento do lactato plasmático e as alterações respiratórias (FURLAN JP, et al., 2019).

\section{Avaliação do lactato e prognóstico}

Lokhandwala S, et al. (2016), analisaram 149 pacientes, em que 42 (28\%) tiveram uma redução menor que $10 \%$ no valor do lactato após medidas terapêuticas, 60 pacientes $(40 \%)$ tiveram uma redução de lactato menor que $20 \%$ e 75 pacientes (50\%) tiveram um segundo lactato medido acima de $4 \mathrm{mmol} / \mathrm{L}$. Os resultados demonstraram que a mortalidade hospitalar foi significativamente maior naqueles pacientes que apresentaram o lactato acima de $4 \mathrm{mmol} / \mathrm{l}$ e aqueles com redução menor que $20 \%$, enquanto aqueles com redução menor que 10\%, não atingiu significância.

Segundo Hernández G, et al. (2019), a normalização da perfusão periférica após reanimação inicial foi relacionada à uma menor mortalidade e menor disfunção orgânica. Assim como Promsin $P$, et al. (2019) através de uma revisão sistemática que incluiu 34 estudos observacionais, concluíram que a diminuição nas concentrações de lactato ao longo do tempo está associada a um prognóstico favorável.

Chambers KA, et al. (2018), realizaram um estudo com 338 pacientes que foram divididos da seguinte maneira: (1) grupo composto por 111 pacientes (32,8\%) com concentração sérica de lactato < $2 \mathrm{mmol} / \mathrm{L}$; (2) grupo composto por 96 pacientes (28,4\%) com valores de lactato sérico entre 2 a $4 \mathrm{mmol} / \mathrm{L}$, e por fim; (3) grupo composto por 131 pacientes $(38,8 \%)$ com lactato inicial acima ou igual a $4 \mathrm{mmol} / \mathrm{L}$. Observaram que os pacientes pertencentes ao grupo 2 apresentaram um aumento da gravidade da doença quando comparado ao grupo 1, e o grupo 3 foi o que apresentou maior risco de mortalidade. No entanto, concluíram que o lactato acima de $2 \mathrm{mmol} / \mathrm{L}$ é fator de pior prognóstico no CS.

Em um estudo de 1.135 pacientes com choque séptico que alcançaram a normalização do lactato em $24 \mathrm{~h}$ do reconhecimento do choque, 806 alcançaram a normalização em $6 \mathrm{~h}$, enquanto 329 o fizeram em 6-24 h. A mortalidade em 28 dias nesses grupos foi de 23,4\% (105/806) e 10,2\% (35/329), respectivamente, enquanto que no grupo de pacientes que não alcançou a normalização em $24 \mathrm{~h}$ foi 68,8\% (309/967). Durante o período de ressuscitação inicial, vários fatores influenciam a mortalidade no choque séptico. No entanto, após a reanimação inicial, o lactato passa a ser um fator mais previsível (RYOO SM, et al., 2018).

De acordo com Ryoo SM, et al. (2019) a normalização do lactato de maneira mais rápida foi fator que contribuiu para diminuição dos níveis de mortalidade. Analisaram cerca de 2102 pacientes, sendo que 806 alcançaram normalização do lactato dentro de $6 \mathrm{~h}$, tendo uma taxa de mortalidade menor do que no grupo que 
não atingiu a normalização dentro das $6 \mathrm{~h}$ (13\% vs $26.5 \%$ ). E cerca de 135 pacientes alcançaram a normalização do lactato dentro de $24 \mathrm{~h}$, tendo então uma taxa de mortalidade menor quando comparado aos pacientes que não tiveram a normalização dentro de $24 \mathrm{~h}$ (12,3\% vs.32,0\%).

A melhora da perfusão nos pacientes com CS ocorre concomitante à diminuição dos níveis de lactato, mesmo que haja alterações em variáveis hemodinâmicas. Dessa forma, a melhora dos parâmetros de perfusão dos tecidos está associada a uma redução do lactato (BAKKER J, et al., 2019).

O aumento do nível de lactato deve ser sempre um sinal de alerta para a equipe médica, requerendo, assim, atenção imediata. A primeira linha de avaliação é muito direta, ou seja, quanto maior for o nível de lactato, maior é a urgência daquele paciente (BAKKER J, et al., 2019). Portanto, a diminuição da produção de lactato e a prevenção da hiperlactatemia de longa duração é de extrema importância no reconhecimento precoce de pacientes com alto risco de mortalidade por CS, e dessa forma, faz-se necessário instruir medidas efetivas para que ocorra a redução deste composto para que a sobrevida aumente de maneira significativa (KHODASHAHI R e SARJAMEE S, 2020).

\section{Relação dos níveis de lactato arterial, oxigênio arterial-venoso e dióxido de carbono venoso-arterial; lactato arterial $x$ lactato venoso}

Nos últimos anos, o dióxido de carbono venoso-arterial tem sido utilizado como um marcador alternativo ao lactato para indicação de hipoperfusão tecidual para avaliação do prognóstico mediante ao diagnóstico de CS. A hiperlactatemia combinada com alta concentração de dióxido de carbono resultou em disfunção orgânica mais grave e pior prognóstico, enquanto sua normalização simultânea foi associada a um melhor desfecho clínico (GAO X, et al., 2021).

O lactato arterial periférico é considerado o padrão ouro para medição das concentrações de lactato em pacientes com choque séptico. $\mathrm{O}$ acompanhamento da depuração do lactato que mais se correlaciona com a mortalidade ocorre durante as primeiras seis horas de ressuscitação nos pacientes com CS. Em estudos sobre o monitoramento do lactato arterial e venoso, os níveis de lactato sérico nas amostras venosas foram mais altos do que aqueles obtidos de acessos arteriais, ainda que tenha havido uma correlação linear direta entre as duas concentrações (MAHMOODPOOR A, et al., 2019).

Uma vez que a hiperlactatemia pode ter outras origens, a não ser hipóxica, a relação do oxigênio arterial venoso e dióxido de carbono com os níveis de lactato não devem ser considerados representativos do metabolismo anaeróbio. Em um estudo observacional em UTI, relatou-se, entre pacientes com CS, presença de hiperlactatemia em $65 \%$ deles, porém, somente $75 \%$ destes pacientes apresentaram aumento dos níveis de lactato, comprovando que a hiperlactatemia pode ser decorrente de outras situações, principalmente durante os estágios iniciais do choque (OSPINA-TASCON GA, et al., 2015).

Não existe um acordo universal sobre o uso da amostra de lactato venoso no lugar do lactato arterial, porém defende-se que as amostras de lactato venoso podem ser usadas nas primeiras horas de reanimação em pacientes sépticos. Pesquisadores afirmam que a amostra do lactato arterial possui algumas desvantagens, como a necessidade de maiores habilidades técnicas e o fato de ser mais invasiva, além de ser financeiramente mais cara (MAHMOODPOOR A, et al., 2019).

Estudos recentes mostram que o dióxido de carbono pode ser usado para identificação de perfusão persistente em pacientes ressuscitados com CS. Relatam, ainda, que não foi encontrada vantagem para ressuscitação à base de lactato, quando comparada à ressuscitação guiada pelo oxigênio (OSPINA-TASCON GA, et al., 2015).

Em um estudo realizado com crianças com CS, mostrou-se correlação aceitável entre lactato venoso e arterial nas concentrações abaixo de $2 \mathrm{mmol} / \mathrm{L}$. No entanto, ressalta-se que as amostras de lactato periférico são influenciadas por mudanças de perfusão local e podem apresentar resultados falsamente elevados (MAHMOODPOOR A, et al., 2019).

\section{Escore da área de lactato}

Usado como fator prognóstico independente nos pacientes com CS com alto risco de mortalidade, o escore da área de lactato é a soma da área sob a curva (AUC) dos níveis séricos de lactato. Se apresenta como um 
fator relacionado à gravidade do aumento do nível de lactato na fase inicial do tratamento e sua persistência. Esta variável calcula o tempo de exposição da hiperlactatemia e as concentrações reais de lactato, além de determinar o valor total de produção de lactato nas primeiras 24 horas após o CS. (KHODASHAHI R e SARJAMEE S, 2020; CHEN H, et al. 2021).

Foi realizado um estudo para determinar a eficácia do escore da área de lactato como marcador de prognóstico do CS. Neste estudo, mediu-se os níveis de lactato em série cinco vezes dentro de 12 horas, indicando que a área de lactato no grupo de não sobreviventes, foi significativamente maior. Como houve associação entre o escore da área de lactato e o índice de mortalidade dos pacientes com CS em 28 dias, definiu-se que o escore da área de lactato inicial pode ser usado para controle de prognóstico (KHODASHAHI R e SARJAMEE S, 2020).

\section{Terapêutica}

A correção da hipovolemia e da hipoperfusão tecidual é inicialmente realizada através da administração de fluidos, visando aumentar a oferta de oxigênio aos tecidos devido ao aumento do débito cardíaco. Atualmente, o fluido de escolha são os cristaloides numa dose de $30 \mathrm{~mL} / \mathrm{kg}$. Porém, quando a ressuscitação volêmica com fluidos não é suficiente para manter a pressão arterial média (PAM) acima dos valores de referência, a utilização de vasopressores deve ser iniciada, sendo a norepinefrina a droga de primeira escolha (ROCHA LL, et al., 2014).

Uma meta-análise proposta por Dellinger RP, et al. (2013) incluindo seis ensaios clínicos controlados randomizados, comparou a utilização de norepinefrina versus dopamina como vasopressor de primeira escolha para pacientes em CS. E neste mesmo estudo, concluíram que a dopamina foi associada a um risco maior de morte $(R R=1,2 ; \mathrm{IC} 95 \% 1,01-1,20 ; p=0,035)$ além do maior risco de arritmias cardíacas ( $R R=2,34$, IC $95 \%$ 1,46-3,77; $p=0,001$ ). Portanto, a norepinefrina foi recomendada como vasopressor de escolha, visto que seu uso trouxe mais benefícios.

Segundo as diretrizes da campanha de sobrevivência à sepse, a transfusão sanguínea pode ser realizada quando o objetivo é atingir um hematócrito de, pelo menos, 30\% (ROCHA LL, et al., 2014). Um estudo publicado, realizado por Holst LB, et al. (2014) comparou dois limites diferentes de transfusão de hemoglobina ( $\leq 7,0 \mathrm{~g} / \mathrm{dL}$ e $\leq 9,0 \mathrm{~g} / \mathrm{dL}$ ) em quase 1000 pacientes diagnosticados com choque séptico, e observaram que não houve nenhuma diferença significativa de mortalidade em 90 dias entre os grupos. Além disso, não foi relatada diferença na incidência de eventos isquêmicos cardíacos e não cardíacos.

Ainda segundo o Instituto Latino Americano de Sepse, a utilização de corticoides pode ser feita em pacientes com CS refratário, isso quer dizer que não conseguem manter a pressão arterial média nos valores de referência, mesmo tendo sido utilizados vasopressores e até mesmo feito a ressuscitação volêmica com cristaloides (ILAS, 2015).

\section{CONSIDERAÇÕES FINAIS}

A elevação deste composto advindo do metabolismo anaeróbico da glicose está relacionado com pior prognóstico, ou seja, a taxa de mortalidade é maior nestes pacientes. Além disso, diversos estudos evidenciaram que quanto maior for o nível de lactato, maior é a chance do paciente ter uma má evolução, principalmente quando associado a outros sinais de hipoperfusão, como a diminuição da diurese, presença de livedo reticular e mottling score com pontuação elevada. Por isso, o diagnóstico precoce do choque séptico, em conjunto com as medidas terapêuticas que buscam diminuir os níveis de lactato, devem ser rapidamente instruídas, a fim de minimizar os danos causados pela desregulação do sistema imune em resposta a uma infecção por um hospedeiro, e assim, diminuir a taxa de mortalidade por CS.

\section{REFERÊNCIAS}

1. ARMSTRONG BA, et al. Sepsis and Septic Shock Strategies. Surg Clin N Am, 2017; 1339-1379.

2. BARROS LL, et al. Fatores de risco associados ao agravamento de sepse em pacientes em Unidade de Terapia Intensiva. Cad. Saúde Colet., 2016, Rio de Janeiro, 24 (4): 388-396

3. BAKKER J, et al. Lactate: Where Are We Now? Crit Care Clin, 2019; 36(1): 115-124. 
4. BENETTI M, et al. Cinética de lactato em diferentes intensidades de exercícios e concentrações de oxigênio. Rev Bras Med Esporte, 2000; 6(2): 50-56.

5. CASTRO R, et al. Efects of capillary refll time-vs. lactate-targeted fuid resuscitation on regional, microcirculatory and hypoxia-related perfusion parameters in septic shock: a randomized controlled trial. al. Ann. Intensive Care, 2020; 10(150): 1-9.

6. CHAMBERS KA, et al.Outcomes of severe sepsis and septic shock patients after stratifi cation by initial lactate value. World J Emerg Med, 2018; 9(2): 113-117.

7. CHEN H, et al. Association between normalized lactate load and mortality in patients with septic shock: an analysis of the MIMIC-III database. BMC Anesthesiology, 2021.

8. CUI NA, et al. Prognostic significance of PCT and CRP evaluation for adult ICU patients with sepsis and septic shock: retrospective analysis of 59 cases. J Int Med Res., 2019; 47(4): 1573-1579.

9. DELLINGER RP, et al. Sobrevivendo ao campo de sepse paign: diretrizes internacionais para tratamento de sepse grave e choque séptico: 2012. Crit Care Med., 2013; 41: 580-637.

10. DING X, et al. Early goal-directed and lactate-guided therapy in adult patients with severe sepsis and septic shock: a meta-analysis of randomized controlled trials. J Transl Med, 2018; 16(331):1-14.

11. DUGAS AF, et al. Prevalence and characteristics of nonlactate and lactate expressors in septic shock. Journal of Critical Care, 2012; 27: 344-350.

12. FURLAN JP, et al. Metabolismo do lactato e avaliação de desempenho: dois lados do mesmo processo. Revista Saúde e Pesquisa, 2019; 10(1):171-179.

13. HERNÁNDEZ G, et al. Effect of a Resuscitation Strategy Targeting Peripheral Perfusion Status vs Serum Lactate Levels on 28-Day Mortality Among Patients With Septic Shock. JAMA, 2019; 321(7):654-664.

14. HERNÁNDEZ G, et al. Effects of dexmedetomidine and esmolol on systemic hemodynamics and exogenous lactate clearance in early experimental septic shock. Critical Care, 2016; 20(234):1-10.

15. ILAS. Instituto Latino-Americano de Sepse. Sepse: um problema de saúde pública / Instituto Latino-Americano de Sepse. Brasília: CFM, 2015.

16. JOUFFROY R, et al. Contribution of the Pre-Hospital Blood Lactate Level in the Pre-Hospital Orientation of Septic Shock: The LAPHSUS Study. Turk J Anaesthesiol Reanim; 48(1): 58-61, 2020

17. KATTAN E, et al. A lactate-targeted resuscitation strategy may be associated with higher mortality in patients with septic shock and normal capillary refll time: a post hoc analysis of the ANDROMEDA-SHOCK study. Ann. Intensive Care, 2020; 10(114): 1-9.

18. KHODASHAHIR, SARJAMEE S. Early lactate area scores and serial blood lactate levels as prognostic markers for patients with septic shock: a systematic review. Infectious Diseases, 2020; 0(0): 1-13.

19. LB HN, et al. Inferior versus superior limiar de hemoglobina para transfusão em choque séptico. N Engl J Med., 2014; 371: 1381-91..

20. LEE SG, et al. Prognostic value of lactate levels and lactate clearance in sepsis and septic shock with initial hyperlactatemia. Medicine, 2021; 100: 1-7.

21. LOKHANDWALA S, et al. Absolute lactate value versus relative reduction as a predictor of mortality in severe sepsis and septic shock. Journal of Critical Care, 2016; 1-22.

22. MAHMOODPOOR A, et al. Arterial vs venous lactate: Correlation and predictive value of mortality of patients with sepsis during early resuscitation phase. Journal of Critical Care, 2019; 58: 118-124.

23. NGUYEN HB, et al. Early lactate clearance is associated with improved outcome in severe sepsis and septic shock. Crit Care Med, 2004; 32(8): 1637-1642.

24. $\mathrm{OH} \mathrm{DH}$, et al. Risk factors for mortality in patients with low lactate level and septic shock. Journal of Microbiology, Immunology and Infection, 2017; 52(3): 418-425.

25. OSPINA-TASCÓN GA, et al. Combination of arterial lactate levels and venous-arterial CO2 to arterial-venous O2 content difference ratio as markers of resuscitation in patients with septic shock. Intensive Care Med, 2015; 41: 796805.

26. PORZIO M, et al. Targeting peripheral perfusion versus serum lactate levels in septic shock. Internal and Emergency Medicine, 2019; 14: 999-1001.

27. PROMSINP, et al. Optimal cut-off for hourly lactate reduction in ICU-treated patients with septic shock. Acta Anaesthesiol Scand., 2019; 63(7): 1-10

28. RANZANI OT, et al. Reclassificando o espectro de pacientes sépticos com o uso do lactato: sepse grave, choque críptico, choque vasoplégico e choque séptico. Rev Bras Ter Intensiva, 2013; 25(4):270-278.

29. ROCHA LL, et al. Current concepts on hemodynamic support and therapy in septic shock. Revista Brasileira de Anestesiologia, 2014

30. RODRIGUEZ A, et al. New definition of sepsis and septic shock: What does it give us? Med Intensiva, 2016; 41(1): 3840.

31. RYOO SM, et al. Timing of Repeated Lactate Measurement in Patients With Septic Shock at the Emergency Department. Am J Med Sci, 2018; 356(2):97-102.

32. RYOO SM, et al. Lactate normalization within 6 hours of bundle therapy and 24 hours of delayed achievement were associated with 28-day mortality in septic shock patients. Plos One, 2019

33. RYOO SM, et al. Momento da dosagem repetida de lactato em pacientes com choque séptico no pronto-socorro. Am J Med Sci. 2018; 356: 97-102.

34. SAUGEL B, et al. Advanced Hemodynamic Management in Patients with Septic Shock Biomed Res Int. 2016; 2016: 8268569.

35. YANG H, et al. Potential biomarkers in septic shock besides lactate. Experimental Biology and Medicine, 2020; 0: 17. 